\title{
Critical Role of the Hippocampus in Memory for Elapsed Time
}

\author{
Nathan S. Jacobs, Timothy A. Allen, Natalie Nguyen, and Norbert J. Fortin \\ Center for the Neurobiology of Learning and Memory, Department of Neurobiology and Behavior, University of California, Irvine, California 92697
}

Episodic memory includes information about how long ago specific events occurred. Since most of our experiences have overlapping elements, remembering this temporal context is crucial for distinguishing individual episodes. The discovery of timing signals in hippocampal neurons, including evidence of "time cells" and of gradual changes in ensemble activity over long timescales, strongly suggests that the hippocampus is important for this capacity. However, behavioral evidence that the hippocampus is critical for the memory of elapsed time is lacking. This is possibly because previous studies have used time durations in the range of seconds when assessing hippocampal dependence, a timescale known to require corticostriatal circuits.

Here we developed a nonspatial paradigm to test the hypothesis that the hippocampus is critical for keeping track of elapsed time over several minutes. We report that rats have a robust ability to remember durations at this timescale. We then determined the role of the hippocampus using infusions of fluorophore-conjugated muscimol, a $\mathrm{GABA}_{\mathrm{A}}$ agonist. We found that the hippocampus was essential for discriminating smaller, but not larger, temporal differences (measured in log units), consistent with a role in temporal pattern separation. Importantly, this effect was observed at long (minutes) but not short (seconds) timescales, suggesting an interplay of temporal resolution and timescale in determining hippocampal dependence. These results offer compelling evidence that the hippocampus plays a critical role in remembering how long ago events occurred.

\section{Introduction}

Different episodic memories typically have many overlapping elements, including specific items and locations, but each experience occurs at a unique time. Therefore, our capacity to distinguish episodic memories critically depends on our ability to remember information about the timing of specific events (Tulving, 1972). Although considerable evidence indicates that the hippocampus is important for remembering the order in which events occurred (Eichenbaum et al., 2005; Kesner, 2013), and for associating events across temporal gaps (Wallenstein et al., 1998), its specific contribution to memory for the temporal context of events remains poorly understood. Recent electrophysiological studies have provided new insights into potential mechanisms. Individual hippocampal neurons show robust timing signals during stimulus-free intervals ("time cells"; Pastalkova et al., 2008; MacDonald et al., 2011) and during the presentation of a series of events (Naya and Suzuki, 2011). Additionally, the pattern of activity in hippocampal ensembles changes gradually with time (Manns et al., 2007; Mankin et al., 2012). Collectively, these

\footnotetext{
Received April 18, 2013; revised July 17, 2013; accepted July 19, 2013.

Author contributions: N.S.J., T.A.A., and N.J.F. designed research; N.S.J., T.A.A., and N.N. performed research; N.S.J., T.A.A., and N.J.F. analyzed data; N.S.J., T.A.A., and N.J.F. wrote the paper.

This work was supported by National Science Foundation Award I0S-1150292, and by the University of California, Irvine. We thank Clare Quirk, Leila Feinberg and Nathan Lo for useful comments on the manuscript. We thank Annie Vogel-Ciernia for help with Arc protein labeling, and John Guzowski for assistance with fluorescence imaging.

The authors declare no competing financial interests.

Correspondence should be addressed to Norbert J. Fortin, 106 Bonney Research Laboratory, University of California, Irvine, CA 92697-3800. E-mail: norbert.fortin@uci.edu.

DOI:10.1523/JNEUROSCI.1733-13.2013

Copyright $\odot 2013$ the authors $\quad 0270-6474 / 13 / 3313888-06 \$ 15.00 / 0$
}

findings suggest that a fundamental role of the hippocampus is to provide an internal representation of elapsed time, which may support our capacity to remember the timing of individual events (Itskov et al., 2011; Shapiro, 2011). However, this crucial link has yet to be demonstrated.

Timing abilities have been studied extensively in humans and animals (Buhusi and Meck, 2005), but the evidence regarding the role of the hippocampus is inconclusive. While timing impairments have been reported in patients with medial temporal lobe damage (Richards, 1973; Noulhiane et al., 2007) and in rats with fornix lesions (Meck et al., 1984), the affected regions were not restricted to the hippocampus. In addition, recent evidence suggests that impairments in traditional timing paradigms may result from a deficit in processing task demands rather than a timing deficiency (Kyd et al., 2008). In fact, basic timing ability is either normal or only mildly impaired with hippocampal dysfunction, though more pronounced deficits are observed when timing must be used flexibly (Meck et al., 1984; Dietrich et al., 1997; Jackson et al., 1998; Kyd et al., 2008). Notably, these studies examined short durations $(<40 \mathrm{~s})$, a scale at which timing is thought to be supported by corticostriatal circuits (Buhusi and Meck, 2005; Meck, 2005), suggesting that uncovering the role of the hippocampus will require the use of longer timescales.

Here we developed a novel behavioral paradigm to test the hypothesis that the hippocampus is critical for keeping track of elapsed time over several minutes. We trained rats to use odorinterval associations to indicate how much time had elapsed since a specific event occurred (i.e., since the previous trial). We determined the contribution of the hippocampus across different time durations using localized infusions of fluorophore-conjugated 
A

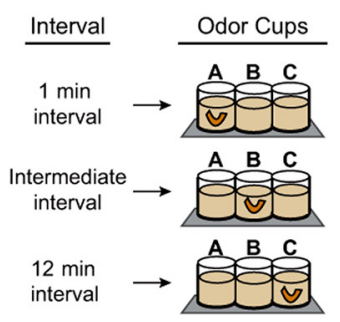

B

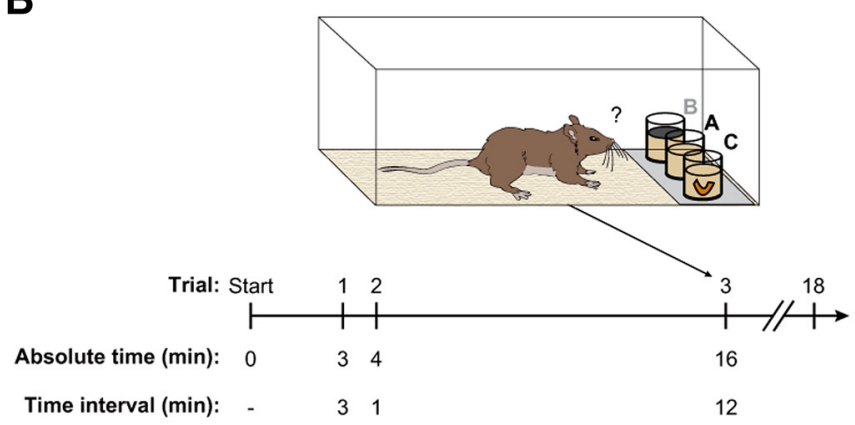

C

\begin{tabular}{ccc} 
Session & Infusion & Condition \\
\hline 1 & FCM & 1min-3min-12min \\
2 & SAL & 1min-3min-12min \\
3 & FCM & 1min-3min-12min \\
4 & SAL & 1min-3min-12min \\
\hline 5 & SAL & 1min-8min-12min \\
6 & FCM & 1min-8min-12min \\
7 & SAL & 1 min-8min-12min \\
8 & FCM & 1 min-8min-12min \\
\hline 9 & FCM & 1 min-1.5min-12min \\
10 & SAL & 1 min-1.5min-12min \\
11 & FCM & 1 min-1.5min-12min \\
12 & SAL & 1 min-1.5min-12min
\end{tabular}

Figure 1. Task and infusion protocol. $A, 0$ dor-interval associations. Rats were trained to indicate how much time had elapsed since the previous trial by selecting the odor associated with that specific time interval: Odor A for a 1 min interval, 0 dor C for a 12 min interval, and Odor B for the variable-length intermediate interval. The correct odor cup contained a cereal reward buried in the sand. $\boldsymbol{B}$, Example session testing intervals of 1,3 , and $12 \mathrm{~min}$. In the depicted example, the rat is offered a choice between 0 dors $A$ and C (i.e., 1 vs 12 min comparison), while 0 dor $B$ is covered. 0 dor Cis rewarded since the previous trial occurred $12 \mathrm{~min}$ before. C, Sample behavioral schedule for one rat. Rats were tested on each condition for four daily sessions, counterbalancing infusions of saline (SAL) and fluorophore-conjugated muscimol (FCM) over consecutive days.

muscimol (FCM; Allen et al., 2008) to temporarily inactivate the hippocampus and verify anatomical spread.

\section{Materials and Methods}

Subjects. Eleven male, 4-month-old Long-Evans rats were used in this study. Rats were experimentally naive and housed individually. Rats were maintained on an inverse $12 \mathrm{~h}$ light/dark cycle and had free access to water. Food was mildly restricted and adjusted for each rat to maintain consistent approach latencies $(0-2 \mathrm{~s})$ during testing. All procedures complied with the Institutional Animal Care and Use Committee.

Behavior. Rats were tested on three conditions, each involving the presentation of three interval durations: (1) 1-3-12 $\mathrm{min}$, (2) 1-8-12 min, or (3) 1-1.5-12 min. Only one condition was tested per session, and one session conducted per day. For a cereal reward, rats were required to determine how much time had elapsed since the previous trial and to select the odor associated with that interval duration, as follows: Odor A for a $1 \mathrm{~min}$ interval, Odor $\mathrm{C}$ for a $12 \mathrm{~min}$ interval, and Odor $\mathrm{B}$ for the intermediate interval $(3,8$, or $1.5 \mathrm{~min}$; Fig. $1 A)$. This design allowed a characterization of the rats' temporal resolution at different timescales.

All testing took place in the rat's home cage (clear polycarbonate; width, $26 \mathrm{~cm}$; length, $48 \mathrm{~cm}$; height, $21 \mathrm{~cm}$ ). Each odor consisted of a household spice (Odor A, $1 \mathrm{~g}$ of sage; Odor B, $1 \mathrm{~g}$ of cinnamon; Odor C, $1 \mathrm{~g}$ of orange peel; "start" odor, $1 \mathrm{~g}$ of cumin) mixed in a clear plastic cup (diameter, $6.35 \mathrm{~cm}$; height, $6.35 \mathrm{~cm}$ ) containing $100 \mathrm{~g}$ of playground sand (Fortin et al., 2002). Cups were secured to a removable acrylic platform $(8.25 \times 24 \mathrm{~cm})$ with Velcro and presented in a single row at the front end of the home cage (Fig. 1B). The location of each odor cup on the platform (left, center, or right) was randomly determined on each trial. Each session began with a start trial, in which a single cumin-scented cup was presented. After rats dug in the cup to uncover the buried cereal reward, the platform was removed and the first interval began. For subsequent trials, rats were presented with Odors A-C. Only the correct odor cup was rewarded, and rats were allowed to dig in only one cup per trial (scored as correct or incorrect). Following this choice, the platform and cups were removed and the next scheduled interval began. Trials were not repeated, and the schedule of trials was not altered during a testing session. All behavioral equipment, including the platform and cups, was removed from the home cage between trials (i.e., during the timing intervals). During intervals, rats were observed grooming, searching, or resting, but no consistent pattern was detected.

To assess performance on each interval comparison separately, a plastic disk covered the sand in one of the two incorrect cups (selected randomly). The covered odor could be smelled, but the rat could not dig in that cup on that trial. This resulted in three types of interval comparisons per condition (e.g., 1 vs $3 \mathrm{~min}, 1$ vs $12 \mathrm{~min}$, and 3 vs $12 \mathrm{~min}$ ). Each session consisted of a randomized schedule of the three time intervals being tested, for a total of 18 trials. As a restriction on true randomization, no more than two consecutive trials involved the same spatial position or interval comparison. All three odors were rewarded at an equal rate, and each interval comparison was tested with equal frequency.

Training and testing occurred over several days. Rats were first trained on the 1-3-12 min condition. After reaching the criterion (75\% correct overall, across 2 consecutive days), rats underwent cannula implantation surgery. Postoperatively, rats were retrained on the 1-3-12 min condition until the criterion was reached again (2-15 sessions; mean, 6.8 sessions). Rats were then tested on the 1-3-12 min condition over four daily sessions [ 2 saline (SAL) sessions and 2 FCM sessions, counterbalanced; Fig. $1 C]$. For both the 1-8-12 min and 1-1.5-12 min conditions, rats were first trained to reach the criterion (1-2 sessions; mean, 1.3 sessions) and then were tested over four daily sessions (2 SAL sessions and 2 FCM sessions, counterbalanced; Fig. $1 C$ ).

Surgery. Rats were anesthetized using isoflurane (1-3\%) to implant four cannulae aimed at the dorsal and ventral hippocampus bilaterally. A midline incision was made in the scalp to expose the skull, and four holes were drilled. Stainless steel guide cannulae $[0.41 \mathrm{~mm}$ outer diameter (o.d.); $0.31 \mathrm{~mm}$ inner diameter (i.d.)] were lowered to $1.8 \mathrm{~mm}$ dorsal to the target coordinates of the infusion cannulae. Implants were secured to the skull with anchor screws and dental cement. Postoperative care was administered for $7 \mathrm{~d}$.

Infusions. The hippocampus was inactivated using fluorophoreconjugated muscimol (FCM) (Allen et al., 2008). Infusions occurred simultaneously at all sites under light isoflurane anesthesia (0.5-2\%). Stainless steel infusion cannulae $(0.25 \mathrm{~mm}$ o.d.; $0.13 \mathrm{~mm}$ i.d. $)$ delivered either SAL $(0.9 \% \mathrm{NaCl}$ in $0.1 \mathrm{M} \mathrm{PBS})$ or FCM $(0.5 \mathrm{mg} / \mathrm{ml}$ in SAL $)$ bilaterally to the dorsal and ventral hippocampus (dorsal: -4.0 anteroposterior, \pm 3.0 mediolateral, -3.8 dorsoventral; ventral: -5.7 anteroposterior, \pm 5.3 mediolateral, -6.4 dorsoventral). For each infusion site, a volume of $0.5 \mu \mathrm{l}$ was infused at a rate of $0.2 \mu \mathrm{l} / \mathrm{min}$. Rats were returned to home cages for $75 \mathrm{~min}$ before testing.

Histology. After a 1 week waiting period, which is sufficient for fluorescence from previous infusions to dissipate (T. A. Allen, L. M. Feinberg, A. Vogel-Ciernia, and N. J. Fortin, unpublished observations), rats were infused with FCM and perfused with $4 \%$ paraformaldehyde 75 min later. Brains were post-fixed for $24 \mathrm{~h}$, cryoprotected ( $30 \% \mathrm{w} / \mathrm{v}$ sucrose), sliced into $50 \mu \mathrm{m}$ coronal sections, mounted, and coverslipped ( $50 \%$ glycerol, $50 \%$ PBS). Sections were imaged using a GE Healthcare Typhoon Trio+ fluorescent scanner with a $532 \mathrm{~nm}$ excitation laser (peak FCM absorption, $543 \mathrm{~nm}$ ) and a $580 \pm 15 \mathrm{~nm}$ emission filter (peak FCM emission, $572 \mathrm{~nm}$ ) at a resolution of $10 \mu \mathrm{m}$. The fluorescence intensity threshold used to indicate areas of tissue inactivation was verified by counterstaining for Arc protein expression, a cellular marker for neural activity (Bramham et al., 2008, immunohistochemistry protocol). For practical reasons, the FCM-Arc relationship was examined in nonbehavioral rats with identical implants and infusions $(n=2)$. 
Statistics. Performance in each condition $(n=6)$ was analyzed separately using repeated-measures ANOVAs with two repeated factors: infusion (SAL, FCM) and interval comparison (e.g., 1 vs $12 \mathrm{~min}, 1$ vs $3 \mathrm{~min}$, and 3 vs $12 \mathrm{~min}$ ). When significant main or interaction effects were observed, post hoc dependent-sample $t$ tests and one-sample $t$ tests (chance $=50 \%$ ) were used to explore the source of the effect. Effects were considered significant at $p<0.05$.

\section{Results}

\section{Localized hippocampal inactivations}

Fluorescence imaging confirmed that a large portion of the rostrocaudal extent of the hippocampus was infused with FCM, and very little FCM spread to other structures (Fig. 2). Fluorescence imaging was performed on eight of the nine behavioral rats; one rat could not be imaged, but his cannulae placements were equivalent to the rats with confirmed hippocampal inactivations. FCM was observed at all four sites in each rat (mean infusion radius, 0.618 $\mathrm{mm}$; SEM, $0.0318 \mathrm{~mm}$; range, 0.434-0.656 $\mathrm{mm}$ ), with comparable spread in the two rats used for immunohistochemistry. Importantly, FCM was colocalized with large reductions in Arc protein expression, verifying the suppression of neural activity (Fig. $2 C, D)$. No statistically significant correlation between infusion extent and task performance was observed.

\section{Discriminating intervals with large temporal differences (1-3-12 min condition)}

Before surgery, rats learned to discriminate three interval durations separated by large temporal differences $(1,3$, and 12 min; log differences, $\geq 0.5$ ), reaching criterion in (mean \pm SD) $21 \pm 7.9$ sessions. Postoperatively, rats continued to perform well following infusions of SAL and FCM (Fig. 3A). Repeated-measures ANOVAs revealed no effect of infusion $\left(F_{(1,5)}=3.890\right.$; $p=0.106)$, interval $\left(F_{(2,10)}=0.416 ; p=\right.$ $0.671)$, or infusion $X$ interval interaction $\left(F_{(2,10)}=0.440 ; p=0.656\right)$. Performance was better than chance on all interval comparisons (SAL vs FCM, all $p$ values $<0.05$ ). These findings demonstrate that general task demands, including the ability to discriminate intervals separated by large temporal differences, do not depend on the hippocampus.

\section{Discriminating intervals with small temporal differences} 1-8-12 min condition

To further examine the rats' temporal resolution, we lengthened the duration of the intermediate interval from 3 to $8 \mathrm{~min}$, while the shorter ( $1 \mathrm{~min})$ and longer (12 min) intervals were held constant (Fig. 3B). This allowed a comparison of performance on
A
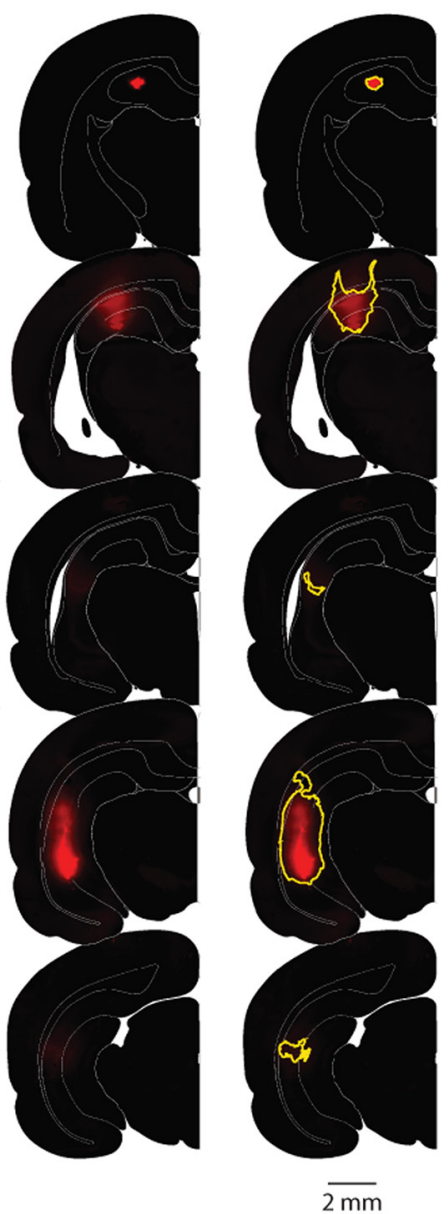

C

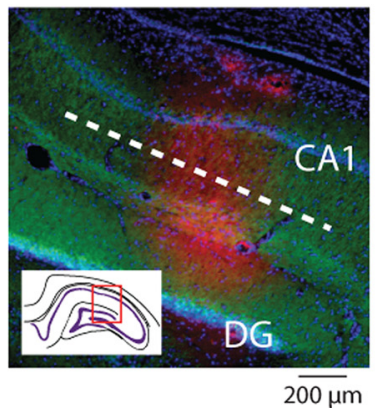

B

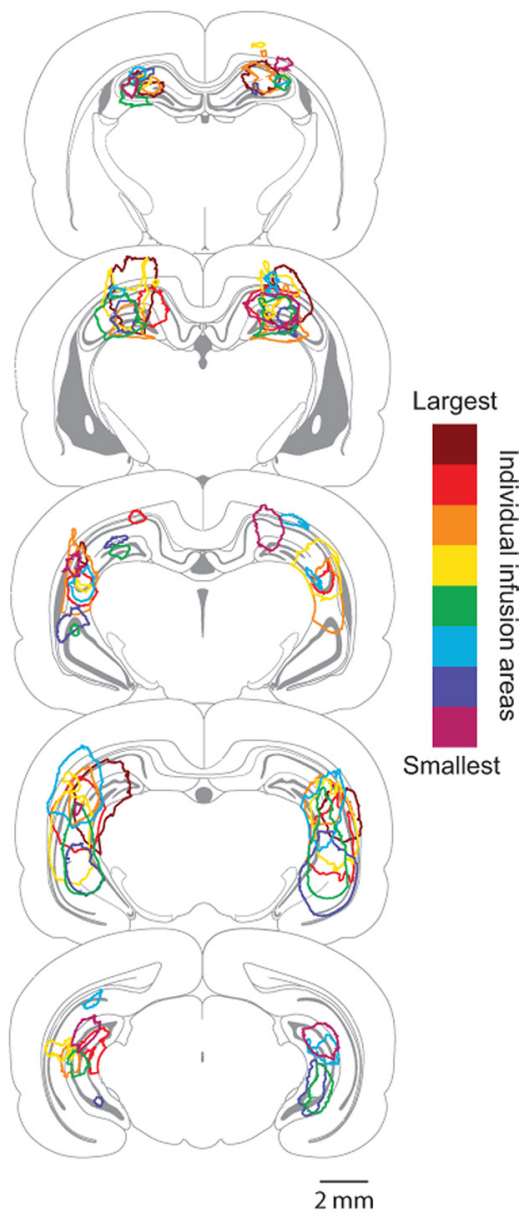

D

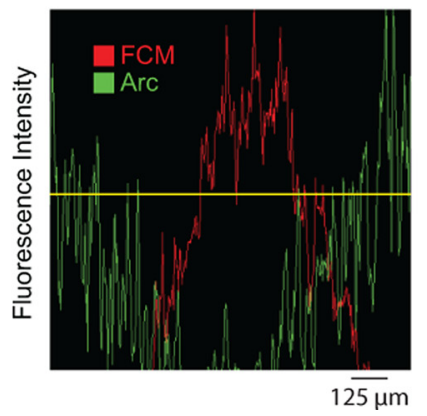

Figure 2. Inactivation of the hippocampus following infusions of FCM. $A$, Area of hippocampal inactivation in a representative subject. Corresponding raw fluorescence images (left column) and estimated maps of inactivation area (right column) are shown at different anteroposterior levels. Inactivation areas were defined as tissue with an estimated FCM concentration $>10 \mu \mathrm{m}$, which has been shown to inactivate neuronal activity in tissue slices (Allen et al., 2008) and substantially inhibit Arc signaling $(\boldsymbol{C}, \boldsymbol{D}) . \boldsymbol{B}$, Overlaid maps of hippocampal inactivation for behavioral rats. The legend color codes the infusion extent for each rat, rank ordered (by square millimeters) from smallest (light purple) to largest (dark red). C, D, The threshold of FCM fluorescence used to determine neural inactivation was verified by counterstaining for Arc protein. $C$, Representative hippocampal tissue slice from a rat infused with FCM (red), and counterstained for Arc protein (green) and DAPI (blue). Fluorescence intensity values from a linear section through the dendritic layers of the representative tissue slice (white dotted line in $C$ ) are plotted to quantify the relationship between FCM and Arc protein (D). Pixels with FCM fluorescence above the threshold (yellow line) were included in the estimated maps of tissue inactivation. DG, Dentate gyrus; $C A 1$, cornu ammonis area 1.

intervals separated by a small temporal difference at a minuteslong scale ( 8 vs $12 \mathrm{~min}$; log difference, 0.2 ), with intervals separated by a large temporal difference ( 1 vs $8 \mathrm{~min}, 1$ vs $12 \mathrm{~min}$; $\log$ difference, $\geq 0.9$ ). Rats continued to perform well on the task after SAL infusions; however, FCM infusions significantly impaired performance. A repeated-measures ANOVA re- 
A

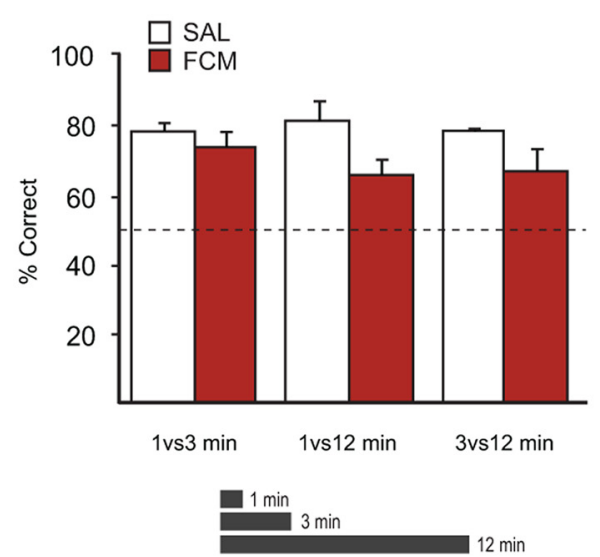

B

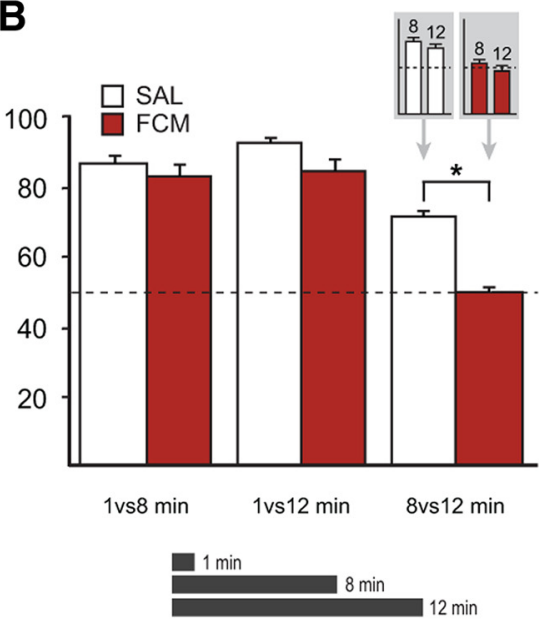

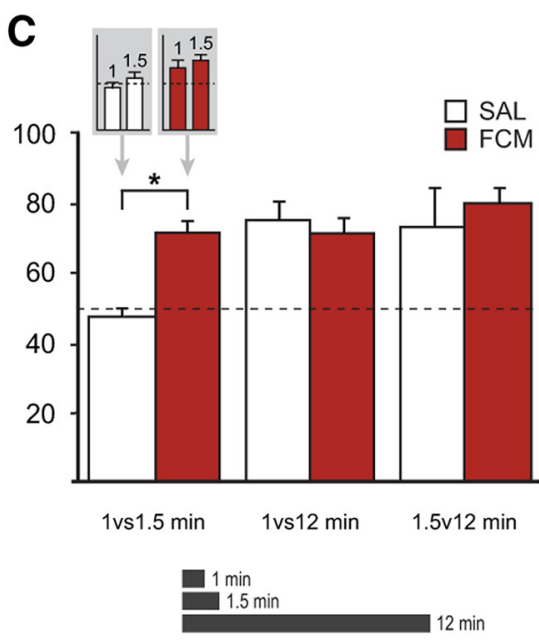

Figure 3. Contribution of the hippocampus to memory for elapsed time. $\boldsymbol{A}$, Hippocampal inactivations did not significantly affect the ability to discriminate intervals separated by large temporal differences (1-3-12 min; log differences, $\geq 0.5$ ). $B$, Hippocampal inactivations selectively impaired the ability to perform fine temporal discriminations between long intervals ( 8 vs 12 min; log difference, 0.2). Insets, Equivalent deficits were observed on 8 and 12 min intervals. C, Hippocampal inactivations significantly facilitated performance on fine temporal discriminations between short intervals (1 vs $1.5 \mathrm{~min}$; log difference, 0.2 ). Insets, Equivalent improvements were observed on 1 and 1.5 min intervals. Accuracy (mean percentage correct \pm SEM) for each interval discrimination was calculated using the combined total of correct and incorrect responses over two saline (SAL) and two fluorophore-conjugated muscimol (FCM) sessions. Dotted line indicates chance accuracy. ${ }^{*} p<0.05$.

vealed a significant effect of infusion $\left(F_{(1,5)}=53.500, p<\right.$ $0.001)$ and interval $\left(F_{(2,10)}=35.829, p<0.001\right)$, but no infusion $\times$ interval interaction $\left(F_{(2,10)}=2.426, p<0.138\right)$.

Post hoc tests showed that FCM infusions impaired the rats' ability to choose the correct odor cup in 8 vs $12 \mathrm{~min}$ trials $\left(t_{\mathrm{SALvsFCM}(5)}=12.448, p<0.001 ; t_{\mathrm{FCMvsChance}(5)}=-0.135, p=\right.$ $0.898)$, with similar deficits found on 8 and 12 min intervals $\left(\mathrm{SAL}_{8 \mathrm{~min}}=77 \%\right.$ correct, $\mathrm{SAL}_{12 \mathrm{~min}}=70 \%$ correct, $t_{(5)}=1.195$, $p=0.286 ; \mathrm{FCM}_{8 \text { min }}=54 \%$ correct, $\mathrm{FCM}_{12 \mathrm{~min}}=45 \%$ correct, $\left.t_{(5)}=1.282, p=0.256\right)$. FCM infusions did not impair performance on 1 vs $8 \mathrm{~min}$ and 1 vs $12 \mathrm{~min}$ comparisons ( 1 vs $8 \mathrm{~min}$ : $t_{\mathrm{SALvSFCM}(5)}=0.572, p=0.592 ; t_{\mathrm{FCMvsChance}(5)}=7.307, p=0.001$; 1 vs 12 min: $t_{\mathrm{SALvFCM}(5)}=1.371, p=0.229 ; t_{\mathrm{FCMvSChance}(5)}=7.238$, $p=0.001)$, suggesting that the deficit was caused by reduced temporal resolution rather than a general performance effect. Thus, the hippocampus is critical for discriminating small, but not large, temporal differences, consistent with a role in temporal pattern separation (Kesner, 2013).

\section{1-1.5-12 min condition}

To determine whether FCM infusions would produce the same pattern of results at short timescales, we reduced the duration of the intermediate interval to $1.5 \mathrm{~min}$ (Fig. 3C). Again, this allowed direct comparison of performance on intervals separated by a small temporal difference, but now at a seconds-long scale (1 vs $1.5 \mathrm{~min}$; log difference, 0.2 ), with intervals separated by a large temporal difference (1 vs $12 \mathrm{~min}, 1.5$ vs $12 \mathrm{~min}$; log difference, $\geq 0.9$ ). Note that the log temporal difference is identical for $1 \mathrm{vs}$ $1.5 \mathrm{~min}$ and 8 vs 12 min comparisons. A repeated-measures ANOVA showed a significant effect of infusion $\left(F_{(1,5)}=20.201\right.$, $p=0.006)$, but no significant effects of interval $\left(F_{(2,10)}=2.017\right.$, $p=0.184)$, nor any infusion $\times$ interval interaction $\left(F_{(2,10)}=\right.$ $1.239, p=0.331)$. Post hoc tests revealed that the effect was specific to the 1 vs $1.5 \mathrm{~min}$ comparison ( $1 \mathrm{vs} 1.5 \mathrm{~min}: t_{\mathrm{SALvSFCM}(5)}=$ $-3.651, p=0.015$; 1 vs $12 \mathrm{~min}: t_{\mathrm{SALvFCM}(5)}=-0.069, p=0.948$; 1.5 vs 12 min: $\left.t_{\mathrm{SALvSFCM}(5)}=-0.786, p=0.467\right)$.

Interestingly, the 1 vs 1.5 min effect was due to better performance in FCM sessions ( $\left.t_{\mathrm{FCMvSChance}(5)}=4.135, p=0.009\right)$ compared with SAL sessions $\left(t_{\text {SALvsChance(5) }}=0.131, p=0.901\right)$, with similar improvements found on 1 and 1.5 min intervals $\left(\mathrm{SAL}_{1 \text { min }}=\right.$ $45 \%$ correct, $\mathrm{SAL}_{1.5 \mathrm{~min}}=55 \%$ correct, $t_{(5)}=0.631, p=0.505$; $\mathrm{FCM}_{1 \min }=66 \%$ correct, $\mathrm{FCM}_{1.5 \mathrm{~min}}=74 \%$ correct, $t_{(5)}=0.690$, $p=0.521)$. This effect was remarkably consistent across rats and is of comparable magnitude to the 8 vs $12 \mathrm{~min}$ impairment mentioned earlier $(20.0 \%$ and $21.5 \%$, respectively). Similar facilitation effects following hippocampal damage have been reported previously (Poldrack and Packard, 2003). These findings suggest that hippocampal processing may be detrimental to the performance of fine temporal discriminations at seconds-long timescales. This is consistent with the hypothesis that timing ability at short timescales primarily depends on regions outside the hippocampus, such as corticostriatal circuits (Buhusi and Meck, 2005; Meck, 2005). Importantly, the data also suggest that the impairment on the 8 vs 12 min comparison described above (Fig. 3B) cannot simply be attributed to task difficulty.

\section{Confounding strategies}

We tested the possibility that nontemporal strategies could have supported performance in the task, and our analyses confirmed that this is not the case. Performance on control trials, in which no reward was given, was not significantly different than on rewarded trials $\left(t_{(4)}=1.327, p=0.255\right)$, indicating that rats could not detect the hidden reward. In addition, there were no consistent response patterns to specific odors or locations.

\section{Discussion}

We developed a behavioral task to investigate the capacity of rats to remember the timing of events, and determined the contribution of the hippocampus at timescales not previously examined (several minutes). We used localized infusions of FCM to temporarily inactivate the hippocampus, confirming specificity using both FCM imaging and a marker of neural activity (Arc protein). While hippocampal inactivations did not affect the ability to discriminate intervals separated by large temporal differences in log units $(1,3$, and $12 \mathrm{~min}$; log differences, $\geq 0.5)$, they produced significant effects when temporal resolution demands were increased. Specifically, hippocampal inactivations severely im- 


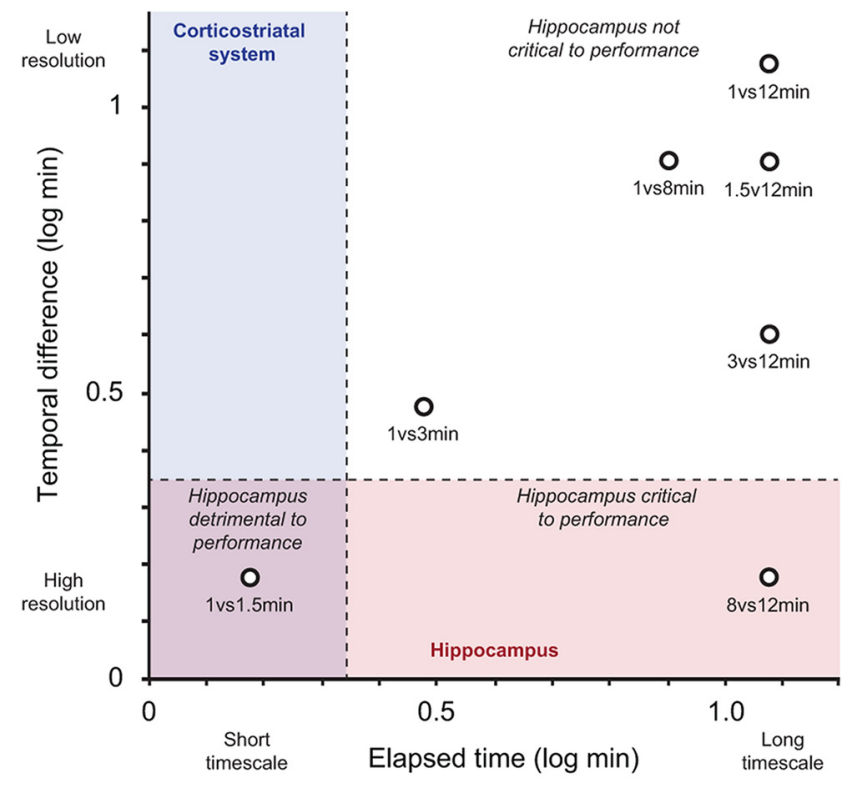

Figure 4. Theoretical interaction of temporal resolution and timescale characterizing the involvement of the hippocampus in memory for elapsed time. Each interval discrimination tested is plotted according to its corresponding temporal difference ( $y$-axis) and timescale $(x$-axis). Areas of the graph thought to engage the hippocampus (high temporal resolution) or corticostriatal circuits (short timescales) are shaded in red and blue, respectively (purple shading denotes overlap). Our data suggest that the hippocampus is critical when the temporal resolution demand is high and the timescale is long (red quadrant, lower right). However, hippocampal involvement at short timescales is thought to interfere with the corticostriatal system; thus, inactivating the hippocampus leads to an improvement in performance (purple quadrant, lower left). Disrupting the hippocampus does not affect performance on discriminations involving large temporal differences at long timescales (white quadrant, top right).

paired performance on fine temporal discriminations between long intervals ( 8 vs $12 \mathrm{~min}$; log difference, 0.2 ). These findings represent a compelling demonstration that the hippocampus is necessary for remembering the time that has elapsed since an event. Interestingly, performance at the same temporal resolution, but a short timescale ( 1 vs $1.5 \mathrm{~min}$; log difference, 0.2 ), was facilitated by hippocampal inactivations. These results indicate that hippocampal processing is detrimental to performance at short timescales, consistent with a hypothesis of systems competition between timing mechanisms in the hippocampus and other structures (Meck, 2005). Overall, the data suggest that hippocampal processing is beneficial when the temporal resolution demand is high and the timescale is long, but adverse when the temporal resolution demand is high and the timescale is short (Fig. 4).

This pattern of results could not have been produced by nonspecific effects of the infusions (i.e., unrelated to timing ability). First, FCM infusions led to a very specific pattern of results, not a broad impairment across conditions. Second, the data were collected over four counterbalanced sessions (two SAL sessions, two FCM sessions), so the effects cannot be due to aberrant performance on a single session or by a subset of the rats. Third, rats were trained before infusions commenced, ensuring that task requirements were learned. Finally, the nonspatial nature of the task ensured that the impairments produced by hippocampal inactivations could not be attributed to spatial deficits.

\section{The hippocampus and timing}

Our findings are consistent with, and extend, previous studies on the neurobiology of timing. Considerable data suggest that timing ability in the range of seconds critically depends on the stria- tum (Buhusi and Meck, 2005; Meck, 2005). Our facilitation effect of hippocampal inactivations on the 1 vs 1.5 min comparison not only supports the view that the hippocampus is not critical for timing at this scale (Dietrich et al., 1997; Kyd et al., 2008), it suggests that the hippocampus can competitively interact with another timing system (Meck, 2005). Similar competitive interactions have been shown between the hippocampus and striatum in other paradigms (Poldrack and Packard, 2003).

Notably, little is known about the neurobiology of timing intervals in the range of minutes. Evidence from neurological patients suggest a duration-dependent involvement of the medial temporal lobe in timing ability, with normal performance at short intervals (<20 s; Richards, 1973), but progressively more pronounced deficits as the interval duration increases (1-8 min; Richards, 1973; Noulhiane et al., 2007). Our findings further suggest that the hippocampus itself plays a key role.

\section{Timing, time cells, and temporal context coding}

The discovery of time cells, neurons in the hippocampus that are active during specific time segments (Pastalkova et al., 2008; MacDonald et al., 2011), has provided new insight into temporal information processing. Although the paradigms in which time cells have been reported do not have explicit timing requirements, a recent computational model proposes that time cells could serve a time-keeping function that would be accurate for tens of seconds (Itskov et al., 2011). Our findings suggest that time cell activity, and hippocampal activity in general, is not critical for the ability to time intervals at that timescale. Instead, time cell coding may be more important for temporally segregating individual events within the context of an unfolding sequence of events (Wallenstein et al., 1998). This hypothesis is consistent with the recent demonstration of sequence position coding in hippocampal neurons (Naya and Suzuki, 2011) and the critical role of the hippocampus in remembering sequences of events (Fortin et al., 2002; Kesner et al., 2002).

Our observation that hippocampal inactivations impair the ability to perform fine temporal discriminations between longer intervals (minutes) suggests that another form of temporal coding in the hippocampus must support performance at these timescales. One possibility is that the pattern of activity in populations of hippocampal neurons, which has been shown to gradually change over time (Manns et al., 2007; Mankin et al., 2012), could serve as a representation of the time of occurrence of individual episodes. Consistent with our findings, the sensitivity of this computation increases with time, indicating it could be particularly useful when timing intervals in the range of minutes, not seconds. Interestingly, the involvement of the hippocampus at a timescale of minutes may be related to the time it takes to form strong contextual representations (Fanselow, 1990). Theoretically, spatial contextual representations in the hippocampus could selectively contribute to temporal coding at long timescales, while elemental (noncontextual) representations supported by structures outside the hippocampus might contribute to coding short timescales.

\section{Conclusions}

A fundamental feature of episodic memory is that the memory of individual events is tied to their spatial and temporal contexts (Tulving, 1972; Clayton and Dickinson, 1998; Eichenbaum et al., 2005). While it is well established that the hippocampus is critical for the memory of the spatial context of events, the present findings add to a growing body of research showing that the hippocampus is critical for the temporal context as well. More 
specifically, the present study provides convincing evidence that its contribution extends to remembering how long ago specific events occurred, particularly when performing high-resolution temporal discriminations. This temporal resolution hypothesis parallels recent findings that the hippocampus is important for discriminating spatial contexts, but only when the contexts are highly similar (Wiltgen et al., 2010). In summary, we show that the hippocampus plays a critical role in remembering how long ago specific events occurred.

\section{References}

Allen TA, Narayanan NS, Kholodar-Smith DB, Zhao Y, Laubach M, Brown TH (2008) Imaging the spread of reversible brain inactivations using fluorescent muscimol. J Neurosci Methods 171:30-38. CrossRef Medline

Bramham CR, Worley PF, Moore MJ, Guzowski JF (2008) The immediate early gene Arc/Arg3.1: regulation, mechanisms, and function. J Neurosci 28:11760-11767. CrossRef Medline

Buhusi CV, Meck WH (2005) What makes us tick? Functional and neural mechanisms of interval timing. Nat Rev Neurosci 6:755-765. CrossRef Medline

Clayton NS, Dickinson A (1998) Episodic-like memory during cache recovery by scrub jays. Nature 395:272-274. CrossRef Medline

Dietrich A, Allen JD, Bunnell BN (1997) Is the hippocampus involved in temporal discrimination and the memory of short intervals? Int J Neurosci 90:255-269. CrossRef Medline

Eichenbaum H, Fortin NJ, Ergorul C, Wright SP, Agster KL (2005) Episodic recollection in animals: "if it walks like a duck and quacks like a duck ..." Learn Motiv 36:190-207. CrossRef

Fanselow MS (1990) Factors governing one-trial contextual conditioning. Anim Learn Behav 18:264-270. CrossRef

Fortin NJ, Agster KL, Eichenbaum HB (2002) Critical role of the hippocampus in memory for sequences of events. Nat Neurosci 5:458-462. CrossRef Medline

Itskov V, Curto C, Pastalkova E, Buzsáki G (2011) Cell assembly sequences arising from spike threshold adaptation keep track of time in the hippocampus. J Neurosci 31:2828-2834. CrossRef Medline

Jackson PA, Kesner RP, Amann K (1998) Memory for duration: role of hippocampus and medial prefrontal cortex. Neurobiol Learn Mem 70: 328-348. CrossRef Medline

Kesner RP (2013) Role of the hippocampus in mediating interference as measured by pattern separation processes. Behav Processes 93:148-154. CrossRef Medline

Kesner RP, Gilbert PE, Barua LA (2002) The role of the hippocampus in memory for the temporal order of a sequence of odors. Behav Neurosci 116:286-290. CrossRef Medline

Kyd RJ, Pearce JM, Haselgrove M, Amin E, Aggleton JP (2008) The effects of hippocampal system lesions on a novel temporal discrimination task for rats. Behav Brain Res 187:159-171. CrossRef Medline

MacDonald CJ, Lepage KQ, Eden UT, Eichenbaum H (2011) Hippocampal "time cells" bridge the gap in memory for discontiguous events. Neuron 71:737-749. CrossRef Medline

Mankin EA, Sparks FT, Slayyeh B, Sutherland RJ, Leutgeb S, Leutgeb JK (2012) Neuronal code for extended time in the hippocampus. Proc Natl Acad Sci U S A 109:19462-19467. CrossRef Medline

Manns JR, Howard MW, Eichenbaum H (2007) Gradual changes in hippocampal activity support remembering the order of events. Neuron 56 : 530-540. CrossRef Medline

Meck WH (2005) Neuropsychology of timing and time perception. Brain Cogn 58:1-8. CrossRef Medline

Meck WH, Church RM, Olton DS (1984) Hippocampus, time, and memory. Behav Neurosci 98:3-22. CrossRef Medline

Naya Y, Suzuki WA (2011) Integrating what and when across the primate medial temporal lobe. Science 333:773-776. CrossRef Medline

Noulhiane M, Pouthas V, Hasboun D, Baulac M, Samson S (2007) Role of the medial temporal lobe in time estimation in the range of minutes. Neuroreport 18:1035-1038. CrossRef Medline

Pastalkova E, Itskov V, Amarasingham A, Buzsáki G (2008) Internally generated cell assembly sequences in the rat hippocampus. Science 321:13221327. CrossRef Medline

Poldrack RA, Packard MG (2003) Competition among multiple memory systems: converging evidence from animal and human brain studies. Neuropsychologia 41:245-251. CrossRef Medline

Richards W (1973) Time reproductions by H.M. Acta Psychol 37:279-282. CrossRef

Shapiro ML (2011) Memory time. Neuron 71:571-573. CrossRef Medline

Tulving E (1972) Episodic and semantic memory. In: Organization of memory (Tulving E, Donaldson W, eds), pp 381-402. New York: Academic.

Wallenstein GV, Eichenbaum H, Hasselmo ME (1998) The hippocampus as an associator of discontiguous events. Trends Neurosci 21:317-323. CrossRef Medline

Wiltgen BJ, Zhou M, Cai Y, Balaji J, Karlsson MG, Parivash SN, Li W, Silva AJ (2010) The hippocampus plays a selective role in the retrieval of detailed contextual memories. Curr Biol 20:1336-1344. CrossRef Medline 